SUBJECT AREAS:

PRE-CLINICAL STUDIES

PHYSIOLOGY

BIOPHYSICS

TRANSLATIONAL RESEARCH

Received

1 March 2013

Accepted

7 June 2013

Published

4 July 2013

Correspondence and requests for materials should be addressed to Y.M. (yossi.mandel@ gmail.com)

\section{Stimulation: New Approach to Control of Non-Compressible Hemorrhage}

\author{
Yossi Mandel ${ }^{1,2}$, Richard Manivanh'2, Roopa Dalal ${ }^{2}$, Phil Huie ${ }^{1,2}$, Jenny Wang ${ }^{1}$, Mark Brinton' \\ \& Daniel Palanker ${ }^{1,2}$ \\ 'Hansen Experimental Physics Laboratory, Stanford University, Stanford, CA, 94305, USA, ²Department of Ophthalmology, \\ Stanford University, Stanford, CA, 94305, USA.
}

Non-compressible hemorrhage is the most common preventable cause of death on battlefield and in civilian traumatic injuries. We report the use of microsecond pulses of electric current to induce rapid constriction in femoral and mesenteric arteries and veins in rats. Electrically-induced vasoconstriction could be induced in seconds while blood vessels dilated back to their original size within minutes after stimulation. At higher settings, a blood clotting formed, leading to complete and permanent occlusion of the vessels. The latter regime dramatically decreased the bleeding rate in the injured femoral and mesenteric arteries, with a complete hemorrhage arrest achieved within seconds. The average blood loss from the treated femoral artery during the first minute after injury was about 7 times less than that of a non-treated control. This new treatment modality offers a promising approach to non-damaging control of bleeding during surgery, and to efficient hemorrhage arrest in trauma patients.

$\mathrm{T}$ rauma is the leading cause of death among US individuals younger than 44 years. Hemorrhagic shock accounts for 30-40 percent of traumatic mortality ${ }^{1,2}$. Bleeding is also the most common preventable cause of death on battlefield ${ }^{3}$. Applications of tourniquets to compressible hemorrhages ${ }^{4-8}$ caused a marked decrease in limb exsanguinations $s^{3,4,9}$. As a result, according to the US army, hemorrhage not amenable to truncal tourniquets (also called non-compressible hemorrhage) is now the leading cause of preventable death ${ }^{3}$. Part of the noncompressible hemorrhages occur due to bleeding into body cavities (such as the abdomen or chest), while others are caused by wounds in the junction between the trunk and the limbs or neck. The latter ones, called junctional hemorrhages, are recognized as a care gap, and those of the pelvic, buttock and groin area are of highest prevalence ${ }^{10}$. Though Combat Gauze ${ }^{\mathrm{TM}}$ is endorsed by the US Army for bleeding care in areas not amenable to a tourniquet, it is often ineffective in junctional hemorrhages such as groin, gluteal, axilla, shoulder and others ${ }^{9,4,3,10}$. A novel mechanical compressing device, the Combat Ready Clamp, was recently introduced into the US Army ${ }^{3,11}$, but has not yet been proven clinically. This device cannot be applied to wounds of the head, neck, abdomen and chest.

Effective prevention of blood loss in the pre-hospital arena offers the best opportunity to save soldiers with non-compressible injuries ${ }^{12}$, therefore major efforts are undertaken to develop technologies for this unmet need. In early $70 \mathrm{~s}$, it was demonstrated that thrombosis can be induced in a clamped blood vessel by minutes-long application of direct electric current ${ }^{13-15}$. However, associated thermal damage precluded the use of this technology in clinical practice. Reduction in blood perfusion during electro-chemotherapy was also noted previously, and it was found to enhance the antitumor effect of the chemotherapy ${ }^{16,17}$. More recently, constriction of blood vessels and thrombosis without thermal damage have been achieved with short ( $\mu s-m s)$ electric pulses ${ }^{18}$. However, these techniques have not been characterized in mammals, nor have they been evaluated for clinical use in various bleeding scenarios.

Recently we described significant decrease in blood loss from liver injury in rabbits treated by sub-millisecond electrical pulses ${ }^{19}$. The current study evaluates the effect of microsecond pulses on blood vessels in two areas nonamenable to truncal tourniquets: the groin area (femoral) and the abdominal cavity (mesenteric). We demonstrate significant vasoconstriction and decrease in blood loss following injury of these blood vessels. These results indicate a possibility of controlling non-compressible hemorrhage using non-thermal pulsed electrical stimulation. 

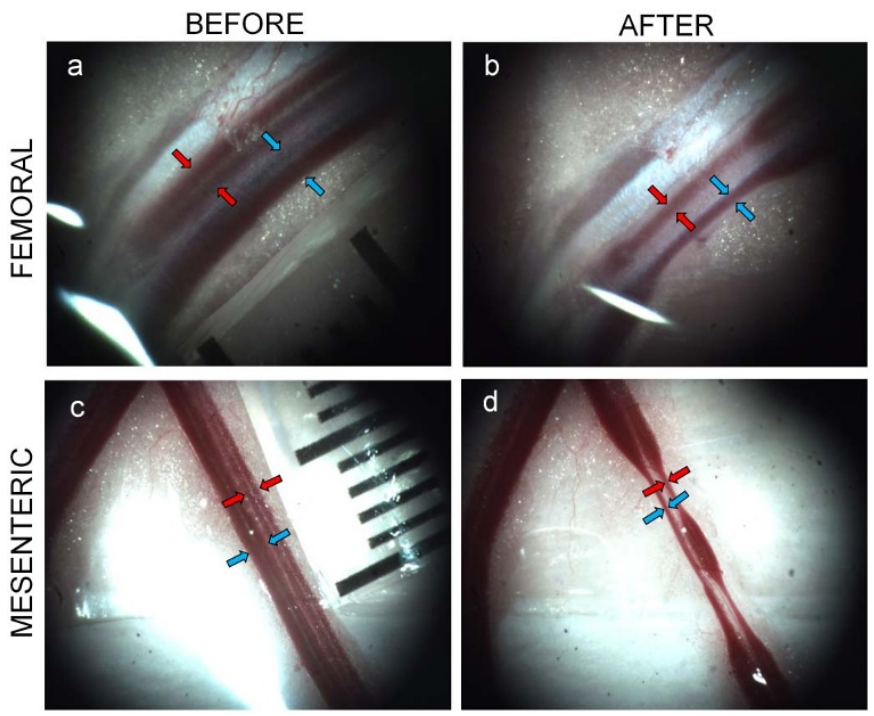

Figure $1 \mid$ Localized vasoconstriction in arteries and veins. Femoral vessels before (a) and after (b) stimulation with $1 \mu$ s/phase pulses of $250 \mathrm{~V}$ at $10 \mathrm{~Hz}$ repetition rate for 30 seconds. Mesenteric vessels before (c) and after (d) stimulation with $1 \mu$ s pulses of $80 \mathrm{~V}$ at $1 \mathrm{~Hz}$ for 30 seconds. Lumens of the femoral and mesenteric arteries and veins are indicated by the red and blue arrows, respectively.

\section{Results}

Constriction of femoral and mesenteric blood vessels. Stimulation current was applied to the exposed blood vessel via $2 \mathrm{~mm}$ diameter pipette electrode filled with saline, while a large pad return electrode was applied to skin on the back side on the animal via conductive gel.
Biphasic (symmetric, anodic-first square) pulses of electric current with duration of $1 \mu$ s per phase, amplitude of $250 \mathrm{~V}$ and repetition rate of $10 \mathrm{~Hz}$ caused, within seconds, a very pronounced local constriction of both femoral (Figure 1a,b) and mesenteric (Figure 1c,d) arteries and veins. Extent of the constriction of femoral vessels in response to 10 seconds long stimulation at $1 \mathrm{~Hz}$ repetition rate is plotted in Figure 2, as a function of stimulus amplitude and duration. Vasoconstriction increased with higher pulse amplitude and longer duration for both arteries and veins. For all pulse durations tested, vessels diameter decreased with increasing pulse amplitude along a sigmoid curve, having a response threshold on the lower end, and reaching a minimum size of about $20-25 \%$ of the original diameter on the high end (Figure 2). Strength-duration dependence of the $25 \%$ and $50 \%$ constriction thresholds could be approximated by a power dependence $t^{-a}$, where $a$ was approximately 0.3 for femoral arteries and veins (Figure 3). For all pulse durations, lower voltage was required to reach similar constriction in arteries, compared to veins, although the difference decreased at longer durations.

Extent of vasoconstriction increased not only with the stimulus amplitude but also with the pulse repetition rate, for both the arteries (Figure $4 \mathrm{a}$ ) and veins (Figure $4 \mathrm{~b}$ ). In this set of measurements, stimulation was applied during 2 minutes, but the vessels constricted to the minimum diameter within about a minute. After the end of stimulation, the blood vessel slowly dilated back to its original width within about 10 minutes (Figure 4 ). The recovery time to $90 \%$ of the original diameter increased with pulse repetition rate. For example, for $1 \mathrm{~Hz}$ it was about 4 minutes, while for $10^{3} \mathrm{~Hz}$ it was about 8 minutes.

Response of blood vessels to continuous stimulation was measured during 10 minute intervals, with the frequency increasing with each step, as shown in Figure 5. Again, the vessels reached the minimum size at each particular frequency within about a minute, and then
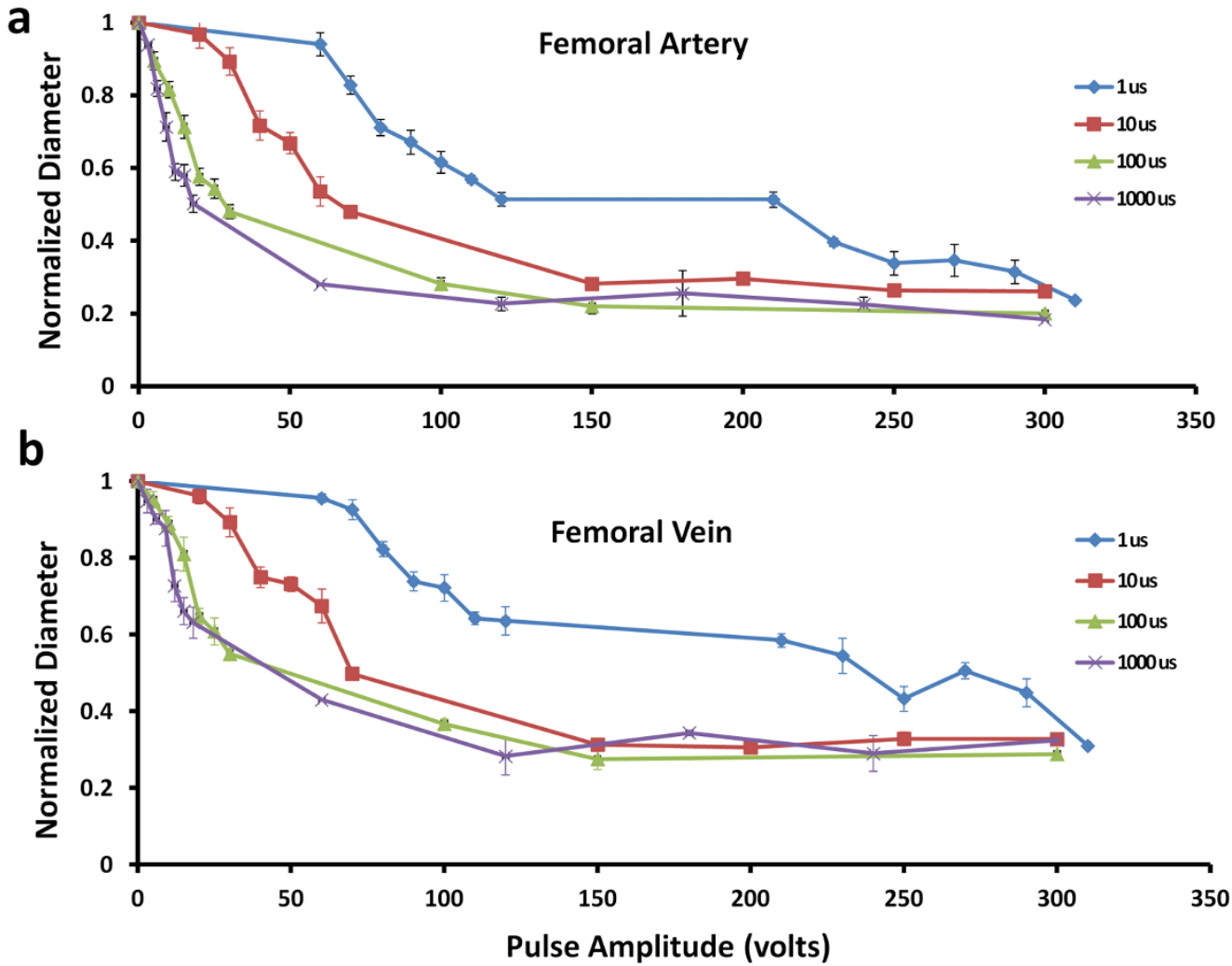

Figure $2 \mid$ Normalized diameter of the femoral arteries (a) and veins (b) in response to electrical stimulation with various amplitudes and pulse durations. Vessels sizes were measured after each stimulation session of 10 seconds in duration. Stimulation pulses were applied at $1 \mathrm{~Hz}$. Vessels were allowed to recover for 20 minutes between the sessions. 


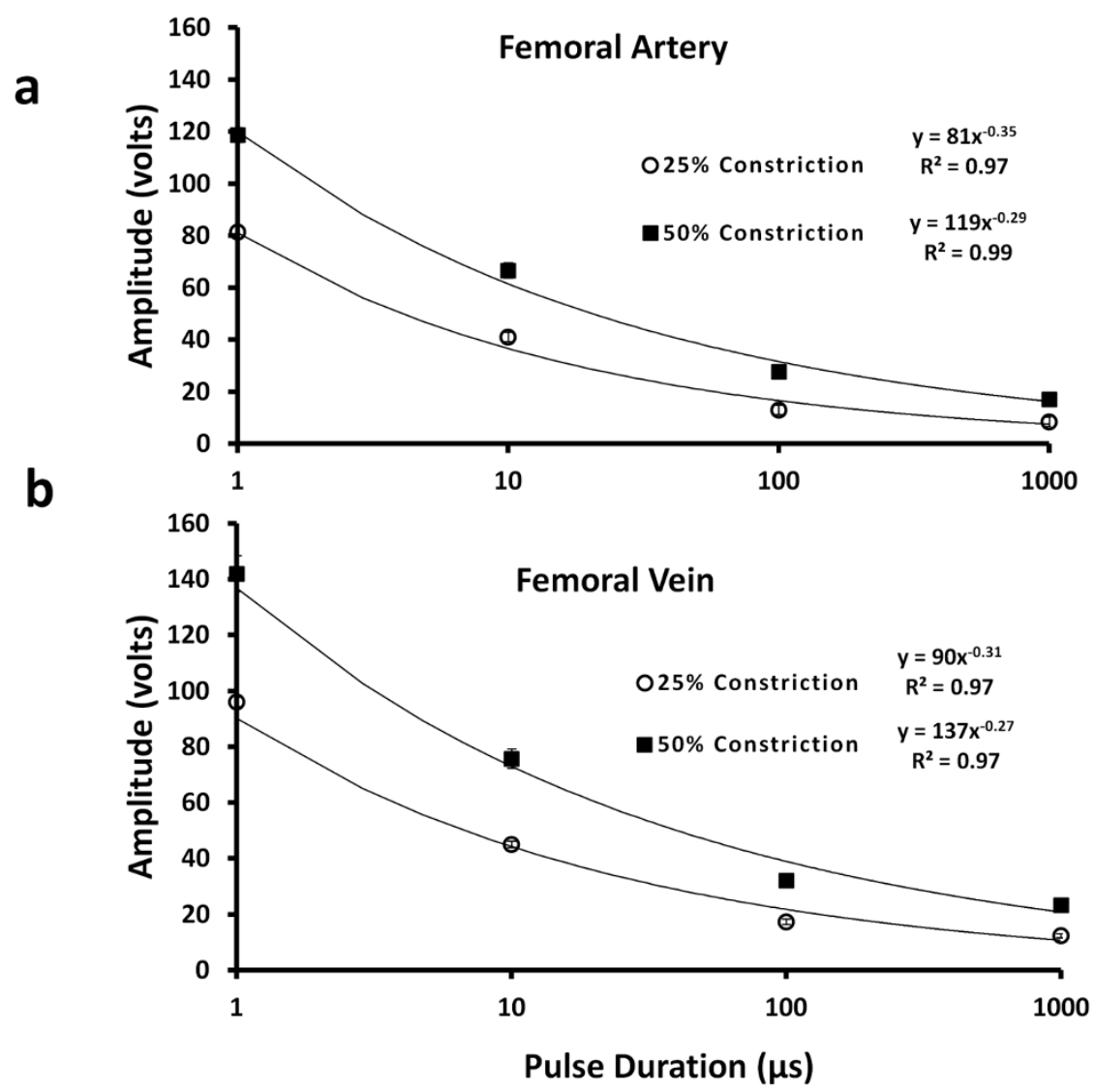

Figure $3 \mid$ Peak voltage required to induce $25 \%$ and $50 \%$ constriction $\left(V_{25}\right.$ and $\left.V_{50}\right)$ with pulse durations of $1,10,100$, and $1000 \mu$ s/phase $(M E A N+/-$ $\mathrm{SE}, \mathbf{n}=6$ ). Analytical fit demonstrated that $\mathrm{V}_{50}$ and $\mathrm{V}_{25}$ thresholds for both types of blood vessels scale with pulse duration as a power function of approximately $\sim t^{-0.3}$.

remained at approximately steady state, with the extent of constriction dependent on the repetition rate. Vasoconstriction was stronger in arteries than in veins for each pulse frequency with both transient (Figure 4) and continuous (Figure 5) stimulation, and the difference was more pronounced at higher repetition rates. Maximum response to continuous stimulation (Figure 5) was smaller than to the transient stimulation regime (Figure 4), especially at higher repetition rates.

Mesenteric blood vessels (Figure 6) had similar kind of response to that of the femoral arteries and veins. For the same pulse parameters, the extent of vasoconstriction in mesenteric arteries was higher than in femoral arteries (Figure 2), and the difference increased with larger amplitudes. For example, with $1 \mu$ s pulses at $200 \mathrm{~V}$ mesenteric arteries constricted by $76 \%$, compared to $49 \%$ reduction in femoral arteries. Mesenteric veins constricted more than the femoral veins at low amplitudes, while this ratio reversed at higher amplitudes.

Hemorrhage control during vascular injury. Complete cut of a femoral artery represents a model of traumatic injury leading to profound loss of blood by the animal. Applying $100 \mu$ s pulses of $150 \mathrm{~V}$ (corresponding to $75 \%$ constriction at $1 \mathrm{~Hz}$ repetition rate) at a repetition rate of $10 \mathrm{~Hz}$ for 30 seconds rapidly decreased the bleeding rate. In all 6 cases treated with this regime, a nearly complete hemorrhage arrest has been achieved within a few seconds. The average blood loss from the femoral artery measured during 30 seconds of treatment and 30 seconds after that was about 7 times less than that of a non-treated control ( 0.14 vs. $1.05 \mathrm{ml}, \mathrm{p}=0.001)$ (Figure 7). In all untreated animals, bleeding still continued after the 1 minute-long blood collection, and the animal died within minutes if bleeding was not mechanically stopped at the end of the measurements. When treated with pulse amplitude of $30 \mathrm{~V}$ at $1 \mathrm{~Hz}$ (corresponding to $50 \%$ constriction threshold), there was no complete hemorrhage arrest, and therefore reduction in blood loss was less pronounced: ( $0.35 \mathrm{vs} 1.05 \mathrm{ml}, \mathrm{p}=0.005)$, as shown in Figure 7). Strong decrease in blood loss was also observed in the severed mesenteric arteries treated with $100 \mu$ s pulses of $40 \mathrm{~V}$ at $1 \mathrm{~Hz}$ (corresponding to $75 \%$ constriction threshold), as shown in Figure 7.

Finite element computational modeling of the resistive heating during the maximum exposure $(150 \mathrm{~V}, 100 \mu \mathrm{s} /$ phase, $10 \mathrm{~Hz}, 30 \mathrm{~s})$ demonstrated that the peak temperature rise at the end of the treatment was between $2.3^{\circ} \mathrm{C}$ with full blood vessel perfusion and $2.9^{\circ} \mathrm{C}$, with no blood perfusion (see Supplementary Figure $1 \mathrm{~b}$ ). This estimate indicates that even the most intense regime of hemorrhage control did not involve thermal damage to the treated vessels. Modeling of resistive heating for reversible constriction $(80 \mathrm{~V}, 1 \mu \mathrm{s} /$ phase, $10 \mathrm{~Hz}, 2 \mathrm{~min}$ ) showed a temperature rise of less than $0.02^{\circ} \mathrm{C}$, indicating that the mechanism of vasoconstriction is not thermal.

Histological findings. Histological sections of the treated and nontreated femoral arteries are shown in Figure 8. Figure 8a shows a cross-section of a femoral artery following complete vessel dissection and 30 seconds-long treatment with $100 \mu$ s/phase pulses of $150 \mathrm{~V}$ at a repetition rate of $10 \mathrm{~Hz}$. For comparison, an untreated control tissue from the other leg is shown in Figure $8 \mathrm{~b}$. Treatment caused complete occlusion of the vessel and cessation of bleeding within a few seconds. Upon euthanasia and tissue fixation the vessels dilated somewhat, compared to the most constricted state because of smooth muscle relaxation. In the middle of a circular lumen one can see an acute blood clot attached to the endothelium (solid arrow). The width of the blood clot illustrates the size of the blood vessel in its 

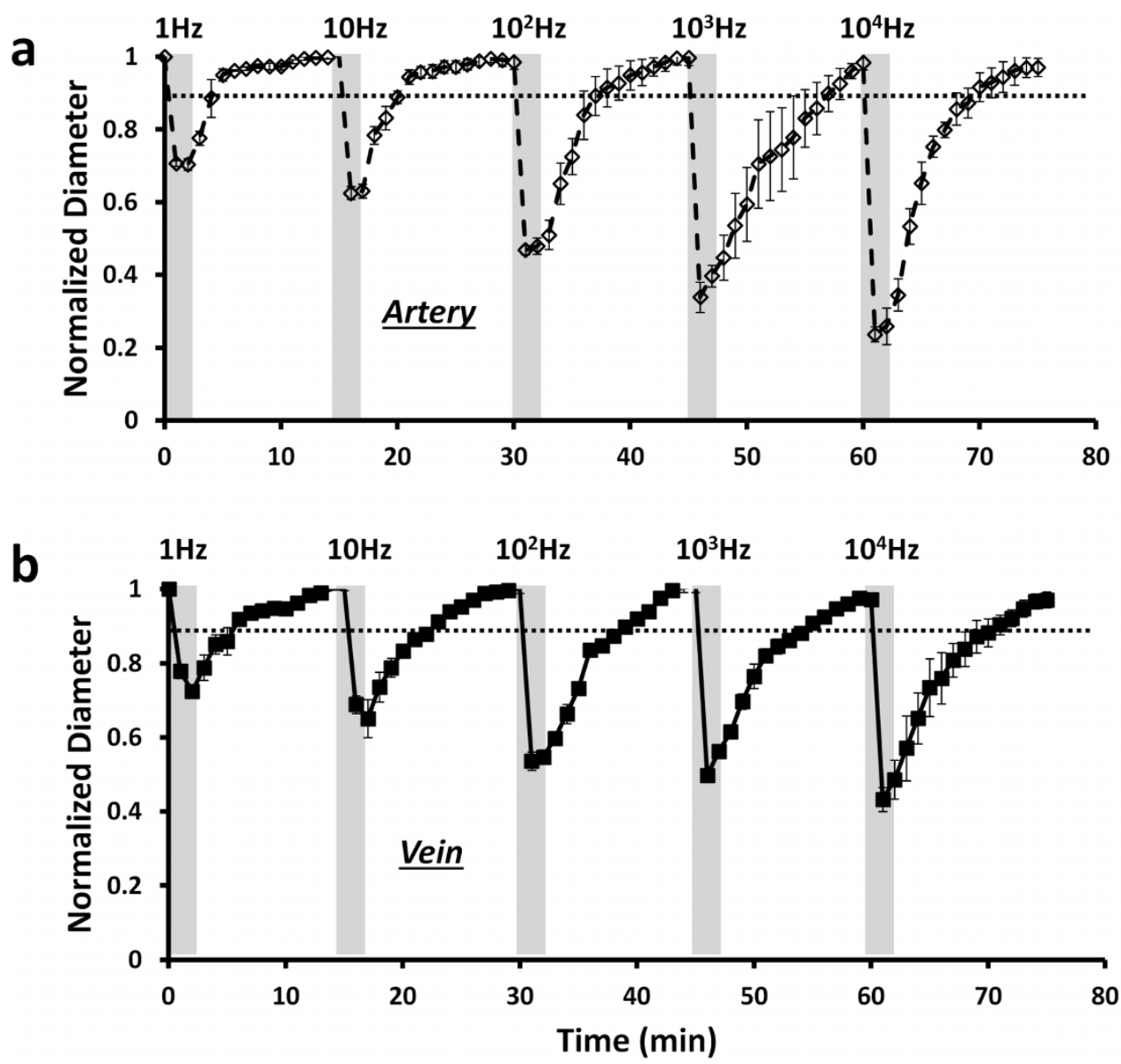

Figure $4 \mid$ Dynamics of the vasoconstriction and recovery of a femoral artery (a) and vein (b) following stimulation with various repetition rates. A single site on the vessel was stimulated at $\mathrm{V}_{25}(80 \mathrm{~V})$ with $1 \mu$ s pulses for 2 minutes and allowed to recover without stimulation during 13 minutes. Repetition rate increased from 1 to $10^{4} \mathrm{~Hz}$ with increments of a factor of 10 .

constricted state, prior to excision and fixation. The smooth muscle contraction is evident by circular appearance of the vessel and by dense folding of the internal elastic lamina (dashed arrow). The endothelium, media and adventitia of the blood vessel appear unaffected by the treatment.

\section{Discussion}

Microsecond electrical pulses can induce vasoconstriction within a few seconds, in both arteries and veins. Upon termination of stimulation, the blood vessels dilate back to their original size within a few minutes. This reversible vasoconstriction can be repeated, and it does not seem to involve tissue damage. Upon stronger stimulation, a permanent blood clot may form, completely blocking the lumen of the blood vessel. Both the reversible vasoconstriction and irreversible clotting offer a powerful approach to hemorrhage control in noncompressible wounds. The extent of perfusion can be controlled by varying the amplitude, pulse duration, and pulse repetition rate. Since the flow rate in a cylindrical pipe is proportional to the fourth power of the diameter (Poiseuille's equation ${ }^{20}$ ), even small constriction of the blood vessel should significantly reduce the flow.

Electrically induced vasoconstriction could result from several effects: stimulation of the sympathetic innervations of the blood vessels and direct stimulation of the smooth muscle ${ }^{21-25}$. We couldn't find reports of a similarly profound pharmacological vasoconstriction - down to almost complete obstruction. This suggests that electrical pulses can induce much stronger contraction of the smooth muscles than pharmacological agents. Interestingly, similar extent of electrically-induced vasoconstriction has been observed in-vitro, with modulation by pulse amplitude, duration and repetition rate $^{23-25}$. Formation of the blood clot may result from localized vascular stasis or a response to injury of the vascular endothelium ${ }^{18,26,27}$.

Histological evaluation of the tissue up to 3.5 hours after vasoconstriction revealed no obvious damage. However, a longer follow-up is required to detect potential development of the inflammatory tissue response, apoptotic effects or other long-term manifestations of mild tissue damage. Long term injury to smooth muscle in the blood vessels was observed one week following exposure to high electric field in rats ${ }^{28,29}$. Injury to vascular endothelium can further enhance the blood clotting and thrombosis ${ }^{30}$.

Conventional thermal coagulation of blood vessels typically requires tens of Watts of power delivered by electrocautery ${ }^{31}$ or RF coagulator $^{31-33}$. Such techniques cause significant tissue injury and are not efficient in coagulation of large vessels. Typically, large vessels require mechanical ligation under direct visualization. Newer techniques such as Ligasure can thermally seal larger arteries, but they require bulky power supply, good visualization of the vessel and access to the vessel from all sides for accurate positioning of the surgical probe, all of which prevent the use of this technology in the field ${ }^{33}$. In contrast, the low-power (few $\mathrm{mW}$ ) electrical vasoconstriction helps reducing blood flow without thermal damage to the tissue, and may not require good visualization of the injured vessel for positioning of the tool. Since very low power is required for such stimulation, a small disposable device could be placed in the wounded area to reduce or stop local bleeding. Pulsatile muscle contraction in response to electrical stimulation could be minimized by using higher repetition rates. 

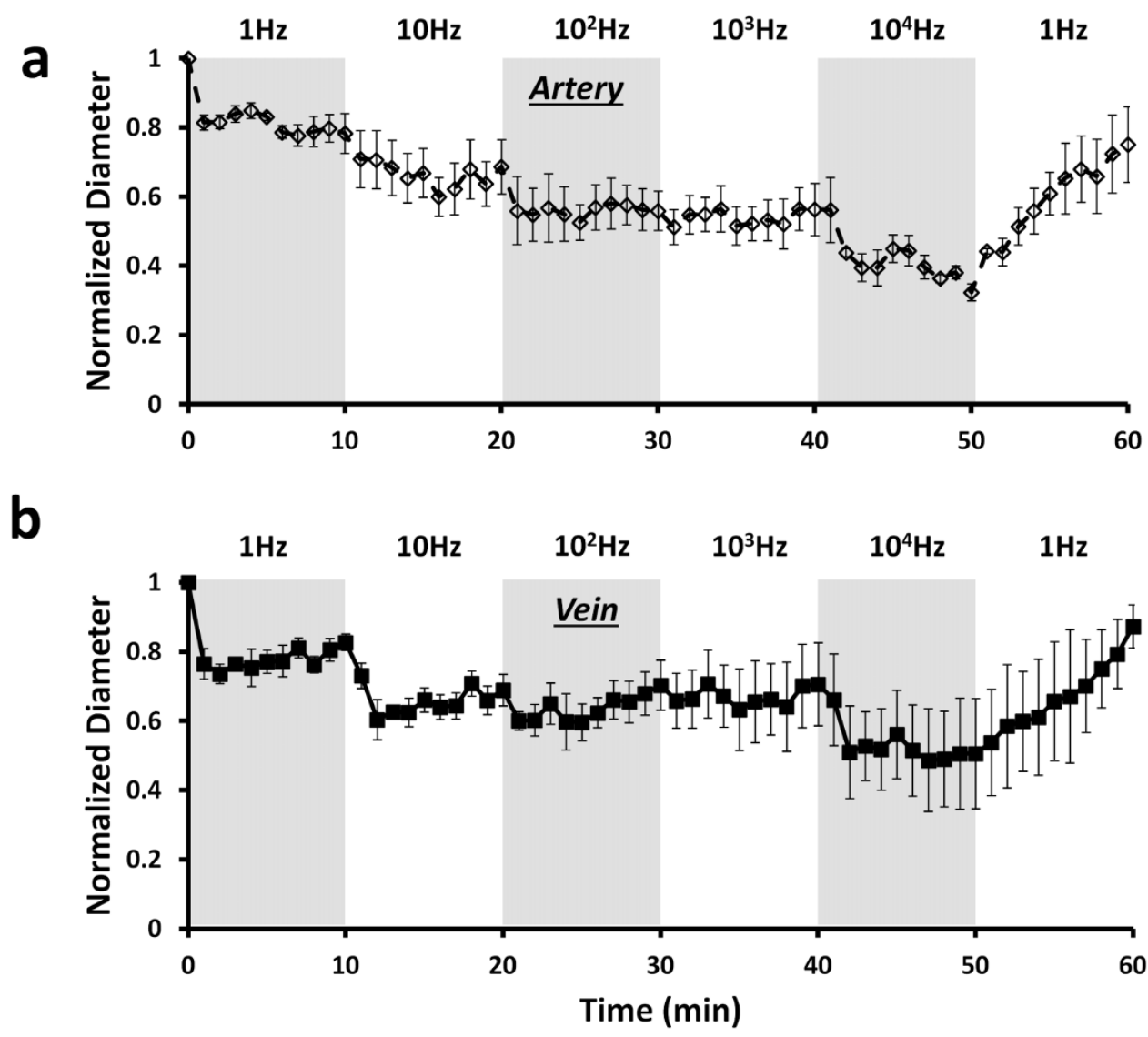

Figure 5 Dynamics of vasoconstriction and recovery of a femoral artery (a) and vein (b) during continuous stimulation with various pulse repetition rates. Blood vessels were stimulated at $\mathrm{V}_{25}(80 \mathrm{~V})$ with $1 \mu$ s pulses for 10 -minute periods at each repetition rate. $(\mathrm{MEAN}+/-\mathrm{SE}, \mathrm{n}=4)$.

In conclusion, electrical stimulation of vasculature by microsecond pulses can be used to control blood perfusion and reduce hemorrhage in non-compressible wounds. Temporary decrease in blood perfusion can be achieved in seconds using the reversible

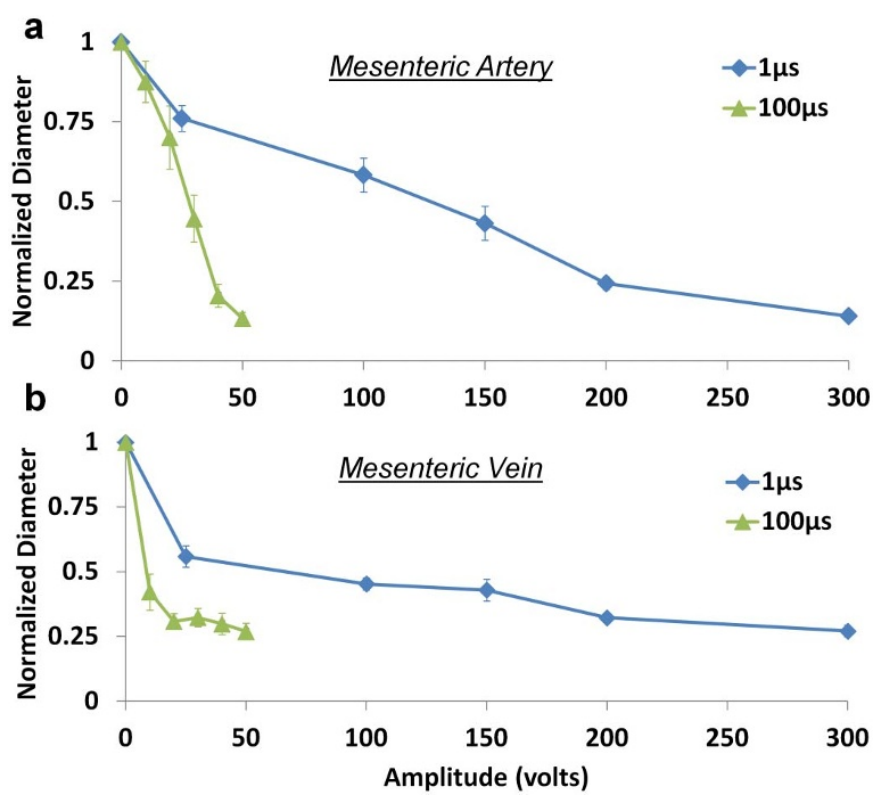

Figure $6 \mid$ Constriction of mesenteric arteries (a) and veins (b) in response to stimulation with pulses of 1 and $100 \mu \mathrm{s} /$ phase in duration. Vessels were stimulated at repetition rate of $1 \mathrm{~Hz}$ during 10 seconds, with 20 minutes recovery between sessions. (MEAN $+/-$ SE, $\mathrm{n}=10$ ). vasoconstriction regime, with vessels dilating back to their original size within minutes after termination of stimulation. This modality could be used for non-damaging hemorrhage control in surgery and during trauma care. Permanent blockage of bleeding is achieved upon vasoconstriction followed by initiation of clotting. For practical use in trauma care and for treatment of the battlefield injuries, a miniature device should be developed capable of delivering pulsed stimulation prior to arrival of the patient to the hospital. Due to low energy requirements a disposable battery-powered device can be just a few millimeters in width, so it can be inserted into the wound to stop local bleeding. Alternatively, a stimulator may remain outside the body, and electric current can be delivered to the area of interest via percutaneous penetrating needle electrodes, similarly to tumor ablation by electroporation [e.g. ${ }^{34}$.

\section{Methods}

Animals. Wild-type Long Evans rats 55-67 days of age were used for all experiments (average weight $275 \mathrm{~g}$ ). Animals were anesthetized with $75 \mathrm{mg} / \mathrm{kg}$ Ketamine $\mathrm{HCl}$ and $5 \mathrm{mg} / \mathrm{kg}$ Xylazine, with additional maintenance half-dose applied every 45 minutes. Buprenorphine $(0.01 \mathrm{mg} / \mathrm{kg})$ and Hartman's Lactated Ringer solution $(114 \mathrm{~mL} / \mathrm{kg} /$ $24 \mathrm{hr} 37^{\circ} \mathrm{C}$ ) were applied sub-cutaneously before experiment for hydration and pain control. All animal care and experiments were carried out in accordance with guidelines for the humane care of animals and were approved by the Stanford Administrative Panel on Laboratory Animal Care.

Electrical stimulation setup. After anesthesia, dorsal side of the animal was shaved and a conductive gel (Signa Gel, Parker Laboratories, Fairfield, NJ, USA) was applied. Animal was placed in supine position on a stainless steel return electrode and internal body temperature was controlled at $37^{\circ} \mathrm{C}$ by a heating pad. Target vessel(s) were dissected from surrounding tissue and allowed to acclimate for 10 minutes before stimulation. A constant drip of $37^{\circ} \mathrm{C}$ Hartman's Lactated Ringer was kept on the exposed site throughout the experiment. Stimulation probe was fabricated from a $1 \mathrm{~mL}$ plastic syringe with $2 \mathrm{~mm}$ diameter opening, filled with normal saline $(0.9 \%$ $\mathrm{NaCl}$ ). A platinum electrode inside the syringe and return pad electrode were connected to a customized biphasic pulse generator and to an oscilloscope 


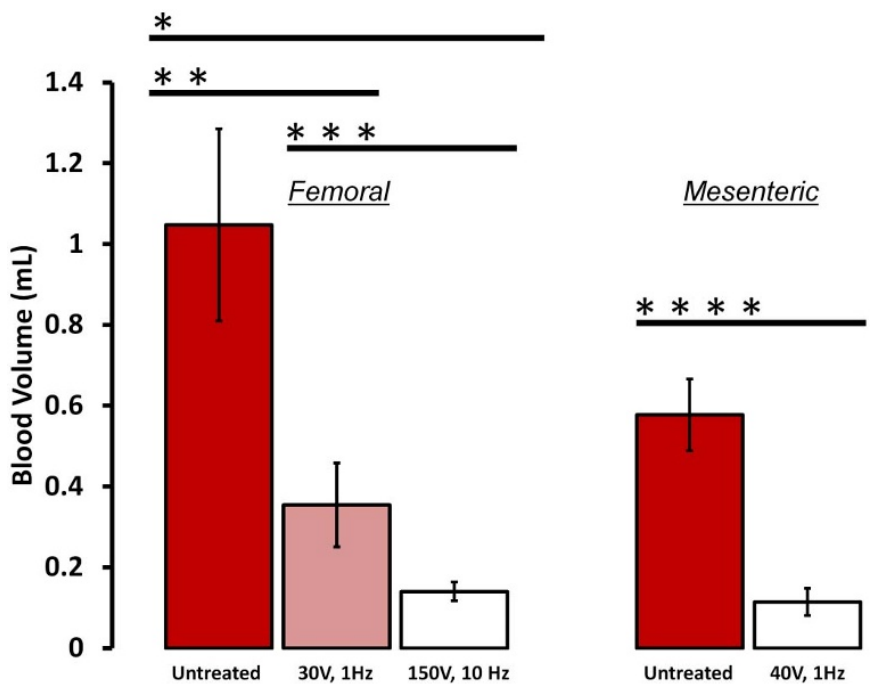

Figure 7 Blood loss following a complete cut of femoral and mesenteric arteries. After cutting, the femoral artery was stimulated for 30 seconds with $100 \mu$ s pulses of $150 \mathrm{~V}$ at $10 \mathrm{~Hz}$ (white bar), or at $30 \mathrm{~V}$ and $1 \mathrm{~Hz}$ (pink bar). Blood was collected during stimulation and for an additional 30 seconds after stimulation. Control vessels were exposed and severed in a similar fashion, and the stimulation probe was placed above the vessel, but no stimulation was applied. Mesenteric vessels were treated with $100 \mu \mathrm{s}$ pulses of $40 \mathrm{~V}$ (right white bar) at $1 \mathrm{~Hz}$ for 30 seconds, or not treated (red bar). In both vessels types, treatment caused decrease or even complete stoppage of bleeding after stimulation, while continuous bleeding was observed in the untreated arteries. Statistical significance of the differences between groups was evaluated using Student t-test: ${ }^{*} \mathrm{p}=0.001,{ }^{* *} \mathrm{p}=$ $0.047 * * * \mathrm{p}=0.005,{ }^{* * * *} \mathrm{p}<0.001$.

(Tektronix, Beaverton, OR, USA). Electric pulses were composed of two square phases of each polarity (positive followed by negative) separated by $10 \mu$ s. Pulse rising time was recorded shorter than $100 \mathrm{~ns}$

Measurements of the constriction threshold. Femoral vessels were stimulated at three sites beginning with the most distal one. Each site was stimulated for 10-seconds at $1 \mathrm{~Hz}$ repetition rate, and left untreated for 20 minutes for recovery. Another stimulation session was then applied to the same site. Mesenteric vessels were stimulated at the second bifurcation from the terminal arteries in intestines, and were treated only once. Images of the blood vessels before and after stimulation were taken with a digital camera (Sentech Inc., TC202USB-A), and the lumen diameters were measured using the ImageJ software.

Measurements of the frequency response. To assess frequency dependence of the vasoconstrictive response, femoral vessels were stimulated at the voltage levels

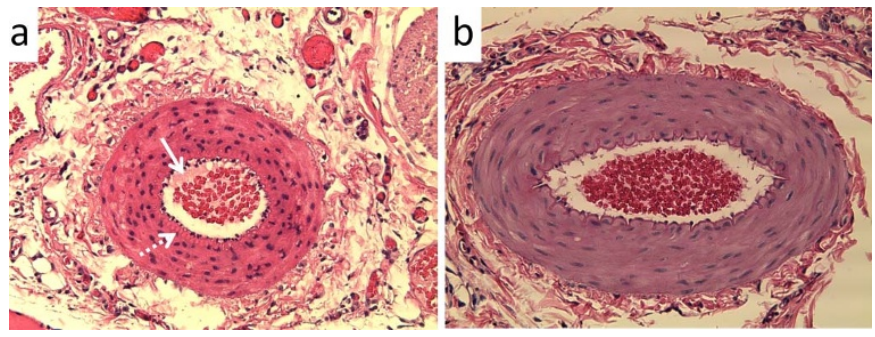

Figure $8 \mid$ Histology of the treated (a) and untreated (b) femoral artery following complete vessel dissection. (a) Femoral artery treated with $100 \mu$ s pulses of $150 \mathrm{~V}$ at $10 \mathrm{~Hz}$ for 30 seconds. Treatment caused complete occlusion of the vessel and termination of hemorrhage within a few seconds. Constricted lumen is filled with acute blood clot attached to the endothelium (solid arrow). Constriction is evident by round shape of the vessel and folding in the internal elastic lamina (dashed arrow). (b) Control femoral artery from the other side of the same animal was cut and left bleeding for 60 seconds. Free flowing blood in the control artery formed a detached clot during sample fixation. corresponding to 25-percent constriction threshold as determined previously for $1 \mathrm{~Hz}$. In one set of experiments the vessel was stimulated for 2-minutes at a certain frequency, and then allowed to recover without stimulation for 13-minutes. This was then followed by stimulation at higher frequency, with the same recovery period (Figure 4). In another set of experiments a continuous stimulation was applied at each frequency for 10 minutes without recovery periods between them. Stimulation probe was removed from the treatment site for a few seconds every minute to capture an image of the tissue. In each step of these experiments repetition rate was increased by a factor of 10 from $1 \mathrm{~Hz}$ to $10 \mathrm{kHz}$, and then back to $1 \mathrm{~Hz}$.

Measurement of the bleeding rate from the injured femoral artery. Femoral artery was exposed and completely cut by surgical scissors. Animals in the treatment group received $100 \mu$ s electric pulses at $150 \mathrm{~V}$ and pulse repetition rate of $100 \mathrm{~Hz}$ for 30 seconds. In a control group electrode was similarly applied near the severed vessel, however no pulses were applied. In both groups hemorrhage volume was measured 1 minute after the vascular injury by soaking a cotton wool near the vessel and weighting the absorbed blood, as previously reported ${ }^{19}$. Statistical significance of the differences between the two groups was evaluated using Student t-test.

Histology. After stimulation the femoral and mesenteric veins and arteries were ligated with 6-0 nylon sutures, and the treated area was dyed with India ink for definitive identification in pathology. Tissue was dissected and fixed in $10 \%$ buffered formalin overnight at room temperature. Tissue was then dehydrated with a graded series of ethanols and fixed in paraffin, sectioned and stained with hematoxylin and eosin $(\mathrm{H} \& \mathrm{E})$

Computational modeling. The tissue temperature rise during electrical stimulation was estimated using an axio-symmetric 2 -dimensional finite element computational model of the electric field and the resulting Joule heating (Comsol Multiphysics 4.1 software, COMSOL inc., USA). The geometry is illustrated in supplemental Figure S1, and the constants are listed in Table S1. In short, the model contained five key components: the muscle tissue, the blood vessel, the plastic pipette, the metal electrode, and the saline in the pipette and in a thin layer above the tissue.

The height of the disk electrode inside the pipette (approximately $2 \mathrm{~mm}$ ) was calibrated with a saline-only model to match the impedance of 619 Ohms measured in a saline-filled petri dish. The tissue resistivity of $300 \mathrm{Ohm}-\mathrm{cm}$ was set in the full computational model to match the total impedance of $1.13 \mathrm{kOhm}$ measured for the experimental setup. After solving the electrostatic field (Poisson) equation which defines distribution of the current density in space, the time-averaged heat source (Joule heating rate per unit volume) was calculated and then added to the Pennes bioheat equation ${ }^{35}$. To account for the blood flow in non-constricted vessel, a perfusion rate inside the blood vessel was set to $10.6 \mathrm{~s}^{-1}$, corresponding to a flow rate of $1 \mathrm{~mL} /$ min through a $1 \mathrm{~mm}$ diameter blood vessel, where the heated length matches the pipette diameter. For treatment resulting in completely occluded vessels, this perfusion was removed.

The room temperature and pipette saline temperature was assumed to be $20^{\circ} \mathrm{C}$, while the saline covering exposed muscle tissue was $37^{\circ} \mathrm{C}$. An equilibration time of $100 \mathrm{~s}$ was used before calculating treatment-induced temperature rise to allow tissue temperature to approach steady state after contact with cooler pipette (see details in Supplementary Material).

1. Center for Disease Control (CDC): Web-based Injury Statistics Query and Reporting System (WISQARS). In: U.S. Department of Health and Human Services, CDC, National Center for Injury Prevention and Control. (2002).

2. Kauvar, D. S., Lefering, R. \& Wade, C. E. Impact of Hemorrhage on Trauma Outcome: An Overview of Epidemiology, Clinical Presentations, and Therapeutic Considerations. J Trauma. 60(6 Suppl), S3-11 (2006).

3. Kragh, J. F. et al. New tourniquet device concepts for battlefield hemorrhage control. US. Army Med. Dep. J. Apr-June, 38-48 (2011).

4. Kragh, J. F. et al. Battle casualty survival with emergency tourniquet use to stop limb bleeding. J. Emerg. Med. 41(6), 590-7 (2011).

5. Kauvar, D. S., Lefering, R. \& Wade, C. E. Impact of hemorrhage on trauma outcome: an overview of epidemiology, clinical presentations, and therapeutic considerations. J. Trauma 60(6 Suppl), S3-11 (2006).

6. McManus, J. G., Eastridge, B. J., Wade, C. E. \& Holcomb, J. B.. Hemorrhage control research on today's battlefield: lessons applied. J. Trauma 62(6 Suppl), S14 (2007).

7. Kelly, J. F. et al. Injury severity and causes of death from Operation Iraqi Freedom and Operation Enduring Freedom: 2003-2004 versus 2006. J. Trauma 64(2 Suppl), S21-6 (2008).

8. Scope, A., Farkash, U., Lynn, M., Abargel, A. \& Eldad, A. Mortality epidemiology in low-intensity warfare: Israel Defense Forces' experience. Injury 32(1), 1-3 (2001).

9. Kragh, J. F. et al. Practical use of emergency tourniquets to stop bleeding in major limb trauma. J. Trauma 64(2 Suppl), S38-49 (2008).

10. Ran, Y. et al. QuikClot Combat Gauze use for hemorrhage control in military trauma: January 2009 Israel Defense Force experience in the Gaza Strip--a preliminary report of 14 cases. Prehosp. Disaster Med. 25(6), 584-8 (2010).

11. US-Army Report. Tactical Combat Casualty Care Guidelines. (2011).

12. Blackbourne, L. H. et al. Decreasing killed in action and died of wounds rates in combat wounded. J. Trauma 69(Suppl 1), S1-4 (2010). 
13. Bourgain, R. H. \& Six, F. A continuous registration method in experimental arterial thrombosis in the rat. Thromb. Res 4(4), 599-607 (1974)

14. Guarini, S. A highly reproducible model of arterial thrombosis in rats. J. Pharmacol. Toxicol. Methods 35(2), 101-5 (1996).

15. Hladovec, J. The effect of some platelet aggregating and potential thrombosispromoting substances on the development of experimental arterial thrombosis. Thromb. Diath. Haemorrh. 29(1), 196-200 (1973).

16. Gehl, A., Skovsgaard, T. \& Mir, L. Vascular reactions to in vivo electroporation: characterization and consequences for drug and gene delivery. Biochimica Biophysica Acta 1569, 51-58 (2002).

17. Sersa, G., Cemazar, M., Parkins, C. S. \& Xchaplin, D. J. Tumour Blood Flow Changes Induced by Application of Electric Pulses. European Journal of Cancer 35(4), 672-677 (1999).

18. Palanker, D., Vankov, A., Freyvert, Y. \& Huie, P. Pulsed electrical stimulation for control of vasculature: temporary vasoconstriction and permanent thrombosis. Bioelectromagnetics 29(2), 100-7 (2008).

19. Mandel, Y. et al. Hemorrhage Control of Liver Injury by Short Electrical Pulses. PLoS ONE 8(1), e49852, doi:10.1371/journal.pone.0049852 (2013).

20. Sutera, S. P. The history of Poiseuille's law. Annu. Rev. Fluid Mech. 25, 1-19 (1993).

21. Atkinson, J. et al. Noradrenaline inhibits vasoconstriction induced by electrical stimulation. Gen. Pharmacol. 18(3), 219-23 (1987).

22. Drummond, P. D. et al. Repeated cycles of electrical stimulation decrease vasoconstriction and axon-reflex vasodilation to noradrenaline in the human forearm. Br. J. Clin. Pharmacol. 64(4), 421-7 (2007)

23. Ferrell, W. R. \& Khoshbaten, A. Responses of blood vessels in the rabbit knee to electrical stimulation of the joint capsule. J. Physiol. 423, 569-78 (1990).

24. Ferrell, W. R., Khoshbaten, A. \& Angerson, W. J. Responses of bone and joint blood vessels in cats and rabbits to electrical stimulation of nerves supplying the knee. J. Physiol. 431, 677-87 (1990).

25. Khoshbaten, A. \& Ferrell, W. R. Nerve-mediated responses of blood vessels in the rabbit knee joint. J. Vasc. Res. 30(2), 102-7 (1993).

26. Darbousset, R., Thomas, G. M. et al. Tissue factor-positive neutrophils bind to injured endothelial wall and initiate thrombus formation. Blood 120(10), 2133-43.

27. Bagot, C. N. \& Arya, R. Virchow and his triad: a question of attribution. Br. J. Haematol. 143(2), 180-90 (2008).

28. Maor, E., Ivorra, A., Leor, J. \& Rubinsky, B. The effect of irreversible electroporation on blood vessels. Technol. Cancer Res. Treat. 6(4), 307-12 (2007).

29. Maor, E., Ivorra, A., Mitchell, J. J. \& Rubinsky, B. Vascular smooth muscle cells ablation with endovascular nonthermal irreversible electroporation. J. Vasc. Interv. Radiol. 21(11), 1708-15 (2010).
30. Geenen, I. L. et al. Coagulation on endothelial cells: the underexposed part of Virchow's Triad. Thromb. Haemost. 108(5), 863-71 (2012).

31. Massarweh, N. N., Cosgriff, N. \& Slakey, D. P. Electrosurgery: history, principles, and current and future uses. J Am Coll Surg 202(3), 520-30 (2006).

32. Mulier, S. Complications of radiofrequency coagulation of liver tumours. British Journal of Surgery. 89(10), 1206-1222 (2002).

33. Alexiou, V. G., Tsitsias, T., Mavros, M. N., Robertson, G. S. \& Pawlik, T. M. Technology-Assisted Versus Clamp-Crush Liver Resection: A Systematic Review and Meta-analysis. Surg Innov. (2012).

34. Cannon, R., Ellis, S., Hayes, D., Narayanan, G. \& Martin, R. C. 2nd. Safety and early efficacy of irreversible electroporation for hepatic tumors in proximity to vitalstructures. J Surg Oncol. doi: 10.1002/jso.23280 (2012).

35. Pennes, H. H. Analysis of tissue and arterial blood temperatures in the resting human forearm. J. Appl. Physiol. 1(2), 93-122 (1948).

\section{Acknowledgments}

Funding was provided by the Government of Israel, Research Grant \# 4440160018. The authors would like to thank LTC Elon Glassberg for a fruitful discussion.

\section{Author contributions}

Conceived the idea and planned the research (YM, DP), performed experiments (YM, RM), thermal model (JW, MRB), histology (RD, PH), wrote the manuscript (YM, DP).

\section{Additional information}

Supplementary information accompanies this paper at http://www.nature.com/ scientificreports

Competing financial interests: The authors declare no competing financial interests.

How to cite this article: Mandel, Y. et al. Vasoconstriction by Electrical Stimulation: New Approach to Control of Non-Compressible Hemorrhage. Sci. Rep. 3, 2111; DOI:10.1038/ srep02111 (2013)

This work is licensed under a Creative Commons AttributionNonCommercial-NoDerivs 3.0 Unported license. To view a copy of this license, visit http://creativecommons.org/licenses/by-nc-nd/3.0 